\title{
Nivel de conocimiento y actitudes de las madres puérperas sobre estimulación temprana en niños menores de un año en el Hospital Nacional Docente Madre-Niño San Bartolomé
}

\author{
Arias- Fernández Sylvia', Chanamé -Ampuero Eva²
}

\section{RESUMEN}

Objetivo: medir el nivel de conocimientos y actitudes de las madres puérperas acerca de la estimulación temprana. Material y métodos: investigación descriptiva, cuantitativa y de corte transversal. La muestra estuvo conformada por 65 madres del Hospital San Bartolomé Madre-Niño. Se construyó dos tipos de cuestionario: el primero, para evaluar el nivel de conocimientos, y estuvo constituido por 15 preguntas; y el segundo, para evaluar el nivel de actitudes, constituido por 10 preguntas. Resultados: el nivel de conocimiento de las madres puérperas es alto en 24 (36,9\%); medio en 29 (44,6\%); y bajo en 12 (18,5\%) de ellas. La actitud hacia la estimulación temprana es favorable en 23 (35\%); indiferente en 28 (43\%) y desfavorable en 14 (22\%). Conclusiones: la mayoría de las madres presentan una actitud indiferente hacia la estimulación temprana, a pesar que tiene un conocimiento de nivel medio. Esta información debe llamar la atención de las enfermeras/os para su intervención oportuna.

Palabras clave: conocimiento, actitudes, estimulación física, desarrollo infantil. Perú. (Fuente DeCs BIREME).

\section{Level of knowledge and attitudes postpartum mothers about early stimulation in children below one year, Hospital Nacional Docente Madre-Niño San Bartolomé}

\begin{abstract}
Objetive: To know the level of knowledge and attitudes of postpartum mothers about early stimulation. Material and methods: Quantitative, descriptive and cross-sectional. With a sample formed by 65 mothers from Hospital San Bartolome Madre -Niño. It was used two types of questionnaires: one to assess the level of knowledge, which was composed of 15 questions and the other to assess the level of attitudes, consisting of 10 questions. Results: : The knowledge's level of postpartum mothers is high with 24 (36.9\%) medium 29 (44.6\%) and low 12 (18.5\%). The attitude towards early stimulation is favorable 23 (35\%), indifferent 28 (43\%) and unfavorable 14 (22\%). Conclusions: The most of the mothers have indifferent attitude toward early stimulation despite of they have regular knowledge. This information must call attention of nurses in order to intervene.
\end{abstract}

Key words: Knowledge, attitudes, physical stimulation, child development. Peru (Source DeCs BIREME).

Licenciada en Enfermería, FAEN-UPCH

Licenciada en Enfermería, docente FAEN-UPCH 


\section{INTRODUCCIÓN}

La estimulación temprana es el conjunto de medios, técnicas y actividades con base científica y aplicada en forma sistémica y secuencial que se emplea en niños desde su nacimiento hasta los seis años, con el objetivo de desarrollar al máximo sus capacidades cognitivas, físicas y psíquicas; permite también, evitar estados no deseados en el desarrollo y ayudar a los padres con eficacia y autonomía, en el cuidado y desarrollo del infante (1).

La estimulación temprana se realiza en los primeros años de vida, y no es necesario que exista alguna deficiencia en el desarrollo intelectual del niño para aplicarla, pero es importante proporcionársela al niño que nace en un ambiente carente de estímulos, para evitar retraso en su desenvolvimiento. Esta serie de acciones no restringe la iniciativa del niño, su curiosidad y su propia necesidad de crecer; por el contrario, busca alcanzar el máximo desarrollo integral logrando lo mejor de sus capacidades mentales, emocionales, sociales y físicas, apoyando el progreso de su inteligencia y personalidad (2).

Para favorecer el óptimo desarrollo del niño, las actividades de estimulación se enfocan en cuatro áreas: lenguaje, esta área abarca tanto el lenguaje verbal, como el no verbal; social, el comportamiento social se refiere a la habilidad del niño para reaccionar frente a las personas y aprender por medio de la imitación; coordinación, esta área comprende las reacciones del niño que requieren coordinación de funciones (óculo - motriz y de adaptación ante los objetos); motora, se refiere al control de la postura y la motricidad (3).

Las posibilidades del desarrollo infantil pueden ser aprovechadas sustancialmente si se trabaja con mediadores innatos, como son los padres de familia y otros adultos que interactúan directamente con el niño en el medio en que nace. La madre es quien, generalmente, prioriza esta mediación y, bien orientada, puede resultar un agente muy significativo en la calidad del desarrollo de sus hijos (4).

El papel de la enfermera, al promover la estimulación temprana al recién nacido, proporciona soporte emocional y educativo a los padres de familia, facilitando el manejo del niño coadyuvando a perder el temor por atenderlo, ya que, a pesar de los logros del niño, aún se presenta una alta incidencia de retraso mental, de audición, ceguera, y de lenguaje que, al momento de identificarlos en el hogar, su impacto es impresionante para los padres, los cuales pasan por conductas de desesperación, hasta llegar a la aceptación del daño de su hijo dándose así su integración al seno familiar en un clima de sobreprotección aun cuando el niño supera su problema los padres continúan percibiendo a su hijo frágil y con necesidad de atenciones especiales (5).

En tal sentido, el profesional de Enfermería que labora en el componente niño, tiene dentro de sus funciones diferentes actividades relacionadas a detectar precozmente alteraciones y/o trastornos en el desarrollo psicomotor, así como establecer coordinaciones pertinentes con el equipo de salud realizando para ello actividades preventivas dirigidas a las madres, con la finalidad de incrementar sus conocimientos sobre estimulación temprana que ayuden al niño alcanzar al máximo sus potencialidades; para ello es importante el apoyo de las madres ya que son ellas las que van a estimular al niño.

Uno de los ejes de la Estrategia Nacional Incluir para Crecer es, precisamente, el desarrollo infantil temprano. Un elemento crucial de estos lineamientos es que buscan articular las atenciones alrededor de las necesidades de los niños desde la gestación hasta los 5 años de vida. El Ministerio de Salud continúa impulsando acciones que contribuyan a mejorar la salud de la mujer, la familia y la niñez. El ministerio viene trabajando desde el Programa de Atención Integral a la Mujer en el desarrollo de la maternidad segura, como una estrategia para mejorar la salud materna. La atención perinatal determina que la embarazada, la parturienta y el recién nacido sean atendidos en el nivel de complejidad que su grado de riesgo requiere $(3,6,7)$.

En las madres puérperas se dan características que muestran una condición única para interesarse y captar conocimientos y contenidos educativos que le facilitarán el cuidado posterior de su hijo. Por lo tanto, el identificar sus conocimientos y actitudes hacia algunos aspectos del cuidado de su niño se hacen relevantes. Considerando esta situación, por lo expuesto anteriormente, se plantea el presente estudio con el objetivo de determinar el nivel de conocimiento y actitudes de las madres puérperas sobre estimulación temprana en el Hospital Nacional Docente Madre-Niño San Bartolomé, en noviembre de 2012.

\section{MATERIAL Y MÉTODOS}

Investigación cuantitativa, descriptiva y transversal. Se realizó en el Hospital Docente Madre-Niño San Bartolomé ubicado en el Cercado de Lima, provincia y departamento de Lima. La población estuvo constituida aproximadamente por 200 madres puérperas que se encontraban hospitalizadas en el Servicio de Puerperio de la institución de salud, por el tiempo determinado de un mes; la muestra es- 
tuvo conformada por 65 madres puérperas que cumplían con los criterios de inclusión. Para la muestra se utilizó la fórmula para población infinita, la técnica de muestreo utilizada fue aleatorio o probabilístico estratificado.

Para la recolección de datos se aplicó a las madres un cuestionario acerca de los conocimientos y actitudes en estimulación temprana, con 15 preguntas y 10 preguntas cerradas, respectivamente. Para evaluar el nivel de conocimientos el puntaje correcto tuvo el valor de 1 y la respuesta incorrecta el valor de 0 . Para medir el nivel de actitudes se utilizó como instrumento de medición la escala de Likert, los puntajes obtenidos en el cuestionario sobre actitud se caracterizaron usando la escala de Estaninos, en tres categorías: actitud favorable, actitud indiferente y actitud desfavorable. Para lo cual se calculó la media (x) y la desviación estándar(s). Para la validez y confiabilidad se aplicó el juicio de expertos y prueba piloto $(\mathrm{p}<0,05$ y alfa $>0,07)$.

Se procedió a revisar y codificar los datos y se introdujeron en una base de datos en el programa Excel. Los resultados fueron analizados porcentualmente.

\section{RESULTADOS}

Gráfico 1. Conocimiento de las madres puérperas sobre las áreas de la estimulación temprana en el Hospital Docente Madre-Niño San Bartolomé. Lima- Perú, 2012

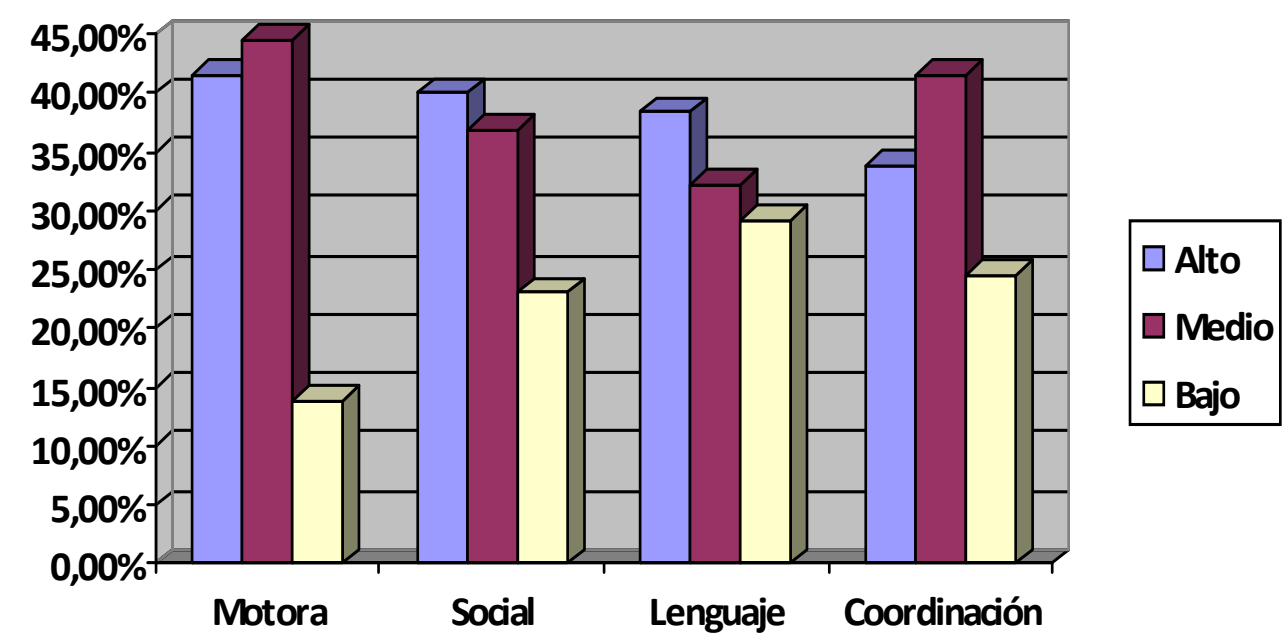

Tabla 1. Nivel de conocimiento de las madres puérperas sobre estimulación temprana en niños menores de un año en el Hospital Docente Madre-Niño San Bartolomé. Lima- Perú, 2012

\begin{tabular}{ccc}
\hline $\begin{array}{c}\text { Nivel de conocimientos sobre } \\
\text { estimulación temprana }\end{array}$ & N & \% \\
\hline Alto & 24 & 36,9 \\
Medio & 29 & 44,6 \\
Bajo & 12 & 18,5 \\
Total & 65 & 100,0 \\
\hline
\end{tabular}

\section{DISCUSIÓN}

En los resultados obtenidos en el gráfico 1, se observa que las madres puérperas encuestadas tienen un conocimiento medio en el área motora con $44,6 \%$ y coordinación 41,5\%; y un nivel alto en el área social (40\%) y lenguaje $(38,4 \%)$. Estos resultados difieren de los encontra-
Tabla 2. .Actitudes de las madres puérperas sobre estimulación temprana en niños menores de un año en el hospital docente madre-niño san Bartolomé. Lima- Perú, 2012

\begin{tabular}{lcc}
\hline $\begin{array}{c}\text { Actitudes sobre } \\
\text { estimulación temprana }\end{array}$ & $\mathbf{N}$ & $\mathbf{\%}$ \\
\hline Desfavorable & 14 & 22,0 \\
Indiferente & 28 & 43,0 \\
Favorable & 23 & 35,0 \\
Total & 65 & 100,0 \\
\hline
\end{tabular}

dos por Valdivia (8) y Chupayo (9). En el primero, el nivel de conocimientos fue alto para el área motora y medio para las otras; en el segundo, fue bajo para todas las áreas. Las madres participantes en este estudio fueron atendidas en una institución de salud del tercer nivel y con anterior experiencia en crianza de hijos; este bagaje influye en su nivel de conocimiento. 
En este punto, cabe resaltar que la madre es quien, generalmente, bien orientada puede resultar un agente muy significativo en la calidad del desarrollo de sus hijos. Las posibilidades del desarrollo infantil pueden ser aprovechadas sustancialmente si se trabaja con mediadores innatos, como son los padres de familia y otros adultos que interactúan directamente con el niño en el medio en que nace (4). Peralta (10) manifiesta que es fundamental reconocer que en las primeras edades se desarrollan y maduran las capacidades fundamentales y prioritarias (20), donde las madres deberían estar en capacidad de estimular todas las áreas y explotar todas las capacidades del niño. En los resultados que se observan sobre el conocimiento global en estimulación temprana (Tabla 1), se evidencia que las madres puérperas encuestadas presentan un nivel de medio con un $44.6 \%$ con resultado similar a los de la investigación realizada por Barzola (11) y Huamanyauri (12). Chupayo, Garrido y Mendoza (9) encontraron un nivel de conocimiento bajo en madres adolescentes. Sin embargo, Valdivia encontró un nivel alto de conocimiento en las madres cuidadoras del programa de Wawasi.

Se sabe, por las investigaciones realizadas en países como Cuba, Ecuador y México, que la capacitación ofrecida a los integrantes del medio más cercano al niño ha tenido un impacto en la familia que se revierte no solo en el propio menor sujeto de la estimulación, sino en cambios conductuales del propio hogar, y que se convierten en comportamientos más o menos perdurables de dicho medio familiar (4). En la presente investigación, el nivel de conocimiento ha sido medio; sin embargo, se debe mantener la capacitación continua para continuar con los conceptos y proporcionar una atención de calidad y oportuna. La actitud es la forma de actuar de una persona, el comportamiento que emplea un individuo para hacer las cosas, también se dice que es un sentimiento a favor o en contra de un objeto social, el cual puede ser una persona, un hecho social o cualquier producto de la actividad humana. Finalmente, se puede concluir que la actitud como una organización duradera de creencias y cogniciones en general, dotada de una carga afectiva a favor o en contra de un objeto definido, que predispone a una acción coherente con las cogniciones y afectos relativos a dicho objeto $(10,13)$.

Referente a la actitud de las madres sobre estimulación temprana (Tabla 2), se observa que $43 \%$ presentó una actitud indiferente. El que la mayoría de las madres presente una actitud indiferente hacia la estimulación temprana puede deberse a la falta de motivación que tuvieron en la etapa prenatal o en consultas de crecimiento y desarrollo con sus otros hijos. Esto indicaría la necesidad de mayor motivación para que asuman la responsabilidad y el cumplimiento de las actividades de estimulación temprana. Tal como refiere Young, las experiencias de aprendizaje del bebé dependerán de cómo la madre intervenga en ellas. La actitud de la madre hará que cada acontecimiento cobre significado por su bebé, y se convierta en un desafío por superar, en una meta por alcanzar o, por el contrario, como una tarea que no tenga sentido intentar (14). De esta manera, si la enfermera incentiva a la madre sobre temas relacionados con la estimulación temprana, y logra captar su interés, entonces la madre se sentirá más segura con los conocimientos adquiridos y ayudará a su bebé a convertirse en una persona con inquietudes por aprender más y a ser capaz de adquirir confianza para enfrentarse a situaciones nuevas de manera independiente.

Bradley (15) refiere que la relación afectiva que se produce entre el niño y la persona que lo atiende, que generalmente es la madre, los sentimientos de seguridad que acompañan la formación de vínculos afectivos adecuados, son la base del funcionamiento psicológico. Es importante la interacción de la madre con los hijos y, el hecho de que se familiaricen con las áreas por estimular para que construyan actitud positiva frente a ellas y así poder ayudar al niño en su desarrollo.

El Ministerio de Salud (MINSA), El Ministerio de Educación (MINEDU), El Ministerio de la Mujer y Desarrollo Social (MIMDES) son los que normativamente deben fomentar y facilitar el acceso a la información sobre el crecimiento, el desarrollo, el aprendizaje infantil, la oportuna y la adecuada educación y estimulación temprana que la familia e instituciones acreditadas pueden brindar a los menores de cinco años $(3,6)$. Los establecimientos y/o instituciones públicas y privadas que realizan actividades de atención y cuidado a la niñez, fomentarán la atención integral, la educación y la promoción de la estimulación temprana dirigida a la madre, el padre y/o responsable de la atención integral de los menores de cinco años. Las actividades que se desarrollan directamente con los niños deben ser organizadas, conducidas y evaluadas por personal capacitado. El equipo interdisciplinario responsable de los servicios de atención integral de la niñez, en el nivel local, promoverá acciones de comunicación educativa que fomenten la promoción de la estimulación prenatal y temprana dirigidas a las mujeres gestantes, las madres con hijos menores de cinco años, los padres y la comunidad en general, contribuyendo a garantizar el desarrollo óptimo infantil en el contexto familiar y comunitario. 
Para tener una idea concreta se puede mencionar que la mayoría de las madres presentan una actitud indiferente hacia la estimulación temprana, a pesar que tienen un conocimiento de nivel medio previo hacia el trato de ellos. Esta información debe llamar la atención de las enfermeras/os para su intervención oportuna.

\section{REFERENCIAS BIBLIOGRÁFICAS}

1. Terré O. V Convención Iberoamericana de Estimulación Prenatal y temprana. Conferencia presentada en: II Congreso Internacional de Estimulación y Psicomotricidad Infantil. JW Marriott Hotel And Stellaris Casino. Perú. 2005. [citado 20 Ene 2010].

2. Romero J. Importancia de La Estimulación Temprana. 2010 [citado 10 Ene 2010]. 5-7. Disponible en: http:/ /portal.unisimonbolivar.edu.co:82/rdigital/psicogente/ index.php/psicogente/article/viewFile/241/230

3. Dirección general de salud de las personas programa mujer-niño sub programa crecimiento y desarrollo. Manual del EEDP. Perú [citado 10 Ene 2010].

4. Martínez F. La Estimulación Temprana: Enfoques, Problemáticas Proyecciones. España; 2006. [citado 10 Ene 2010]. Disponible en: http://www.oei.es/inicial/ articulos/enfoques_estimulacion_temprana.pdf

5. Macias N., Hernández I., Rodríguez A. La enfermera en estimulación temprana del $\mathrm{RN}$ de alto riesgo y Prematuros. México. [citado 6 Feb 2010]. Disponible en: http://www.uasnet.mx/enfermeria/trab04.html

6. Ministerio de Salud. Modelo Integral de Salud. Disponible en: http://www.minsa.gob.pe/portal/ Servicios/SuSaludEsPrimero/MAIS/mais.asp [citado 25 Mar 2010].

7. Banco interamericano de desarrollo. El Perú, primero la infancia. Perú; 2013. [citado 02 Dic 2013]. Disponible en http://blogs.iadb.org/desarrolloinfantil/ 2013/11/06/en-peru-primero-la-infancia/ Valdivia A. Nivel de conocimiento de conocimientos y actitudes sobre la estimulación temprana que tienen las madres cuidadoras de los niños de 1 año de edad del Programa Nacional Wawa Wasi. [Tesis].Cañete: Universidad Nacional Mayor de San Marcos; 2009.
8. Chupayo H., Garrido M. y Mendoza M. Conocimiento sobre estimulación temprana y desarrollo psicomotor de las madres adolescentes y su relación con el nivel de desarrollo psicomotor del lactante. Sociedad de Beneficencia Pública de Lima Metropolitana. [Tesis].Lima; 2007.

9. Peralta, V. Deber o Derecho. [Internet]. Conferencia presentada en el I Simposio Mundial de Educación Preescolar en el siglo XXI. Chile. Disponible:http:// www.oei.es/inicial/articulos/situaciones_educa tivas_desarrollo_integral.pdf [citado 19 Feb 2010].

10. Barzola K. y Camarena M. Nivel de conocimientos y actitudes del padre sobre estimulación temprana en niños menores de cinco anos del Centro Materno Infantil El Progreso Carabayllo. [Tesis].Lima; 2008.

11. Huamanyauri J. Grado de conocimiento de las madres sobre estimulación temprana en los lactantes menores de un año que asisten al componente Niño-Hospital Local de Huaycan Ate-Vitarte. [Tesis].Lima: Universidad Nacional Mayor de San Marcos; 2005.

12. Papalia E. Diane. Psicología del Desarrollo. Onceava edición. México: Editorial Mcgraw Hill; 2009. [citado 9 Ene 2010].

13. Young, K.; Flügel, J. y otros. Psicología de las Actitudes. Buenos Aires: Editorial Paidós SA. [citado 10 Jun 2010].

14. Bradley B. Concepción de la Infancia. Madrid: Alianza editorial; 2006. [citado 18 Jun 2010].

\section{Correspondencia}

Eva Chanamé Ampuero

Universidad Peruana Cayetano Heredia

Dirección: Av. Honorio Delgado 430, Urb. Ingeniería, S.M.P.

Correo electrónico: eva.chaname@upch.pe

Forma de citar este artículo: Arias- Fernández S, Chanamé Ampuero E. Nivel de conocimiento y actitudes de las madres puérperas sobre estimulación temprana en niños menores de un año en el Hospital Nacional Docente Madre-Niño San Bartolomé. Rev. enferm Herediana.2014;7(1):39-43. 